\title{
Variétés de vigne de demain : évolutions attendues, attentes professionnelles et sociétales, challenges
}

\author{
Laurent Audeguin ${ }^{1, a}$, Christophe Sereno ${ }^{1}$, et Olivier Yobrégat ${ }^{2}$ \\ ${ }^{1}$ Institut Français de la Vigne et du vin - Pôle Matériel Végétal, Domaine de l’Espiguette, 30240 Le Grau du Roi, France \\ ${ }^{2}$ Institut Français de la Vigne et du vin - Pôle Sud-ouest, V'innopôle BP. 22, 81310 Lisle sur Tarn, France
}

\begin{abstract}
Résumé. En 2015, le Catalogue Officiel Français comprend 377 variétés tous usages confondus. Chaque année, des variétés nouvelles viennent enrichir cette liste afin d'offrir à la viticulture des outils pour relever les défis qui se présentent à elle. En effet, la prise de conscience du changement climatique, la réduction recherchée des intrants phytosanitaires et la capacité à maintenir la compétitivité des entreprises font naître beaucoup d'attentes en matière de matériel végétal. Depuis les années 1960, la sélection clonale continue d'apporter des réponses (état sanitaire, performances) aux viticulteurs dans la mesure de la diversité naturelle que peuvent offrir les cépages traditionnels. Fertilité, vigueur, taille des grappes et des baies, arômes sont des critères que la sélection a pris en compte et continue d'étudier. Il en est de nouveaux que les conservatoires permettent d'appréhender : tardiveté, maturité polyphénolique, port,... Que faut-il en attendre ? Vis-à-vis du réchauffement climatique, des sélections accumulant moins de sucres tout en permettant une bonne maturation des polyphénols ? Des travaux sont en cours, mais les marges permises par la variabilité naturelle sont réduites et certaines questions devenues primordiales resteront sans doute sans réponses, concernant les grandes préoccupations sanitaires : maladies du bois, mildiou, oïdium, viroses...

Dans ces domaines, la création variétale apparaît comme le seul levier de progrès significatif. Initiée au début des années 2000 par l'INRA et désormais en partenariat avec l'IFV, la création par hybridation de variétés de vigne résistantes au mildiou et à l'oïdium vise un double objectif : durabilité des résistances et qualités agronomiques, technologiques et gustatives. Les premières inscriptions au Catalogue sont prévues fin 2016. On assiste également depuis quelques années à un regain d'intérêt pour des variétés locales. Circuits courts, vins de niches à forte identité, sont autant d'arguments en faveur de la réintroduction de cépages délaissés, moyen idéal pour se démarquer d'une forme de standardisation.

Le paysage composé de nos variétés classiques est donc inéluctablement appelé à évoluer. Sur quel pas de temps, dans quelles conditions ? Difficile à prédire mais il faut se rappeler que les hybrides représentaient la moitié du vignoble français dans les années 1950. Vivement demain?
\end{abstract}

\section{Introduction}

En France, la création de la « Section de Sélection et de Contrôle des Bois et Plants de Vigne » en 1944 par le professeur Jean Branas marque le début de la sélection moderne de la vigne en France (1). En effet, étant donné le mauvais état sanitaire vis-à-vis du court noué du vignoble français à cette époque, la justification de cette démarche s'est rapidement imposée. Elle aboutit dans les années 60 à la publication de la première version du catalogue officiel pour la vigne. Depuis, de nouvelles variétés et clones viennent enrichir régulièrement cette liste grâce au travail conjoint de l'Institut Français de la Vigne et du vin (IFV), des partenaires régionaux de la sélection vigne (au nombre de 33) et de l'INRA. En 2015, le Catalogue français comprend ainsi 377 variétés inscrites et plus de 1200 clones agréés. Aux 346 variétés à raisins (destinées à la cuve, la table, l'agrément, la conserverie), issues de $V$. vinifera ou hybrides interspécifiques, s'ajoutent 31

\footnotetext{
a Auteur de correspondance: Laurent . audeguin@vignevin. com
}

porte-greffes (2). Face aux enjeux que représentent désormais le changement climatique, la réduction des intrants phytosanitaires et la capacité à maintenir la compétitivité des entreprises de la filière vitivinicole française tant sur le marché intérieur qu'au niveau international, l'amélioration continue du matériel végétal constitue un des leviers majeurs de progrès.

\section{Rappel des différents niveaux de conservation des ressources génétiques vigne en France On distingue 3 niveaux complémentaires :}

La collection ampélographique centrale nationale et internationale (INRA, Domaine de Vassal) : établie depuis 1959 à Marseillan-plage (Hérault), elle compte aujourd'hui plus de 7500 génotypes, dont 5500 accessions représentant 2600 variétés traditionnelles de Vitis vinifera. Ses objectifs sont multiples i) Conservation de la diversité génétique la plus étendue possible, tant au niveau français qu'étranger ii) Conservation de gènes d'intérêt (résistances, comportements

This is an Open Access article distributed under the terms of the Creative Commons Attribution License 4.0, which permits unrestricted use, distribution, and reproduction in any medium, provided the original work is properly cited. 


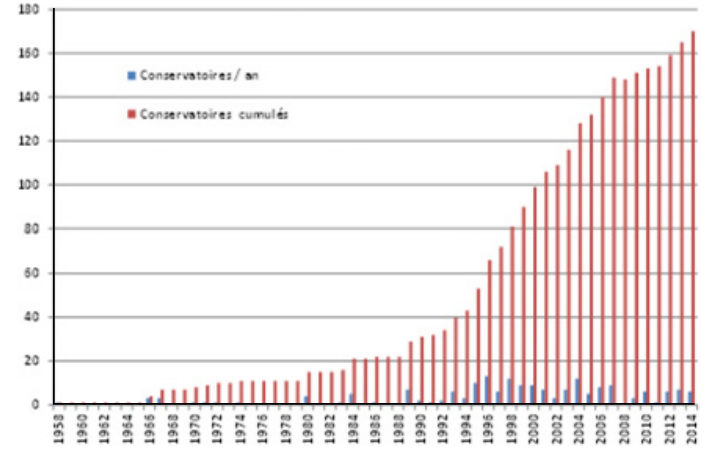

Figure 1. Dynamique d'installation des conservatoires de clones en France - O Yobrégat 2015.

agronomiques, mutations, etc.), iii) Détention de références fiables pour l'identification des variétés (descriptions ampélographiques, herbiers, profils ADN, documents), iv) Fourniture de ressources (recherche, sélection, création variétale, etc.).

Le conservatoire national du matériel initial des clones sélectionnés (IFV, Pôle Matériel Végétal, Domaine de l'Espiguette). Implanté au Grau-du-Roi (Gard), il constitue le centre de sélection de l'Institut Français de la Vigne et du Vin (IFV). Ses missions sont centrales i) Sélection sanitaire (tests ELISA, PCR, indexages), ii) Maintien du matériel initial des clones agréés, iii) Coordination du Réseau des Partenaires de la Sélection (voir ci-dessous) avec l'INRA, iv) Appui aux travaux dans les régions (prospections, expertises, agrément de clones et inscriptions de variétés au Catalogue national), v) Fourniture de matériel végétal à la filière, vi) Pilotage de programmes de recherche (génétique, viroses, etc.). Depuis sa création, plus de 19000 clones y ont été introduits et testés ; environ 5500 y sont actuellement maintenus, représentant près de 500 variétés (raisins de table, de cuve, porte-greffes).

Les conservatoires régionaux de clones au sein du Réseau des partenaires de la sélection vigne. On dénombre aujourd'hui 170 parcelles, représentant près de 130 variétés inscrites au Catalogue national, pour un total d'environ 20000 accessions conservées. Le réseau des Partenaires de la Sélection compte aujourd'hui 33 membres conventionnés autour de l'IFV et de l'INRA. Ces conservatoires ont pour vocation la conservation de la diversité intra-variétale la plus large possible et la présélection de clones.

Inscription de variétés au catalogue officiel. Le catalogue officiel français des variétés de vigne comporte trois listes distinctes : A1 (inscription et classement donnant droit à cultiver la variété), A2 (inscription et diffusion dans l'Union Européenne) et B (inscription et diffusion hors UE). Pour toute nouvelle inscription dans l'une de ces trois catégories, la variété candidate doit être dénommée sans équivoque possible et faire l'objet d'un examen DHS (Distinction, Homogénéité et Stabilité) qui consiste en une description ampélographique détaillée et incontestable. En outre, pour figurer sur les listes A1 ou A2, la variété candidate doit faire l'objet d'un dossier technique qui sera

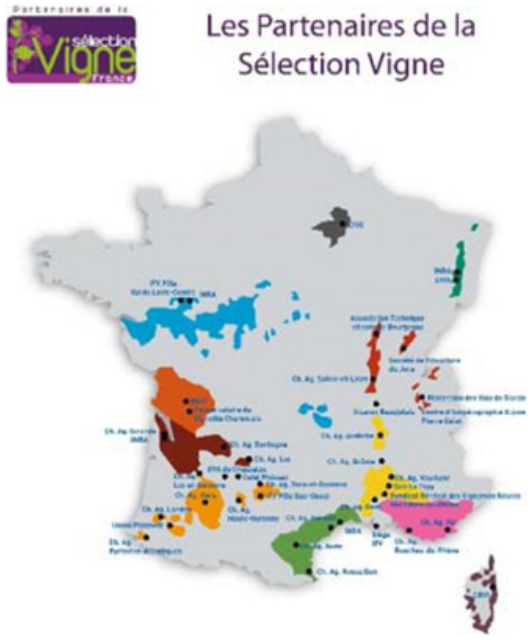

Figure 2. Les partenaires de la sélection vigne. Document IFV.

analysé par la section vigne du Comité Technique Pour la Sélection (CTPS). Deux cas de figure sont alors possibles :

- pour une variété traditionnelle historique déjà référencée en France ou à l'étranger : une étude bibliographique des aptitudes culturales et technologiques peut être jugée suffisante,

- pour une variété d'obtention récente ou pour une variété traditionnelle insuffisamment ou non documentée: une évaluation exhaustive de VATE (Valeur Agronomique Technologique et Environnementale) s'avère nécessaire. Celle-ci nécessite la mise en place de parcelles expérimentales selon un protocole précis (3) : dispositif implanté sur 2 sites distincts, minimum de 3 blocs de 30 souches par dispositif ( 3 blocs de 8 souches pour raisin de table), 5 ans de suivi viticole et 3 ans de vinification et dégustations.

Ce dispositif étant minimal, la section vigne du CTPS réfléchit actuellement à la définition d'une limite maximale (surface) à la VATE.

\section{Le volet intra-variétal, une variable d'ajustement !}

La sélection d'un clone jusqu'à son agrément est un processus long qui se déroule en plusieurs étapes. Ce travail se fait en collaboration avec les partenaires régionaux de la sélection vigne qui constituent un réseau national de sélectionneurs regroupant des Chambres d'Agriculture, des Interprofessions, des Syndicats et des Associations. Ce groupe est animé par l'IFV, dont certains pôles régionaux sont eux-mêmes acteurs de la sélection. Il permet d'échanger sur les problématiques de la sélection, de la conservation des ressources génétiques et de coordonner des expérimentations sur le matériel végétal au niveau national.

Après pré-sélection au vignoble ou dans un conservatoire de clones, les individus jugés intéressants sont introduits au domaine de l'Espiguette pour le dépistage des viroses. C'est la phase de sélection sanitaire au cours 
de laquelle l'indexage (complété de tests ELISA et RTPCR) reste la méthode officielle. Suite à cette première étape, les clones sains sont installés pour expérimentation dans la région d'origine, en collection d'étude, dont le suivi viticole et technologique jusqu'à la dégustation va permettre de définir le profil cultural et technologique de ces clones. C'est la phase de sélection génétique. A l'issue, les clones jugés les plus intéressants en fonction des objectifs de sélection sont alors présentés à la section vigne du CTPS pour agrément (4).

Depuis 2013, la section vigne impose désormais ( $a$ minima pour les variétés disposant déjà d'au moins un clone agréé) 6 répétitions de 5 souches, 5 années de suivi au vignoble, 3 années de vinification suivies de dégustations.

Dans le cas de l'examen d'une nouvelle variété (création variétale ou variété non référencée), l'agrément du clone constitue un cas particulier et repose sur : (a) le dossier génétique, constitué du rapport d'expérimentation VATE établi par l'obtenteur et de l'avis du CTPS Vigne, sur lequel s'appuie le ministère chargé de l'agriculture pour prononcer l'inscription au catalogue ; (b) le dossier sanitaire, constitué des tests de référence sur le matériel d'origine détenu par l'obtenteur.

50 ans de sélection clonale permettent aujourd'hui de mettre en perspectives ces travaux au regard des objectifs poursuivis.

On peut ainsi considérer que la sélection a vécu 3 phases générant 《 3 générations de clones ». Depuis les années 60 jusqu'à la fin des années 70 , la $1^{\text {ère }}$ génération représente des clones sélectionnés sur la base de leur bon état sanitaire et de leur potentiel de production plutôt élevé (Cabernet-Sauvignon 15, Chardonnay 76, 96, Grenache N 70 , pour ne citer qu'eux).

Les années 80 ont vu l'émergence de sélections dites 《 qualitatives » dont le potentiel de production s'est avéré limité (Chardonnay 548, Syrah 470... .).

Actuellement, les objectifs sont de compléter la gamme de clones disponibles, autant que faire se peut, c'est-à-dire dans la mesure où la diversité intra-variétale d'un cépage donné offre cette possibilité.

Cette notion de complément de gamme s'accompagne également de la nécessité de répondre à des problématiques spécifiques à chaque cépage. On peut ainsi mentionner le renouvellement des clones historiques de CabernetSauvignon 191, 337 et 341 porteurs d'enroulement type 2 (GLRaV-2) qui, avec le concours de la Chambre d'Agriculture de la Gironde a débouché sur 2 nouveaux clones, 1124 issu du clone 191 assaini par microgreffage d'apex et le clone 1125, issu des collections de l'INRA et proche en termes de performances du clone 337.

Les études sur le dépérissement de la Syrah ayant mis en évidence un facteur clonal déterminant dans la sensibilité au problème, de nouveaux clones originaires du conservatoire de Mercurol suivi par la Chambre d'Agriculture de la Drôme ont été récemment agréés. Ils portent les numéros 1140, 1141 et 1188 .

Actuellement, les recherches et les attentes sont à la fois précises et diverses : port érigé, moindre compacité des grappes, tardiveté et accumulation de sucres modérée, acidité plus élevé, potentiel aromatique.

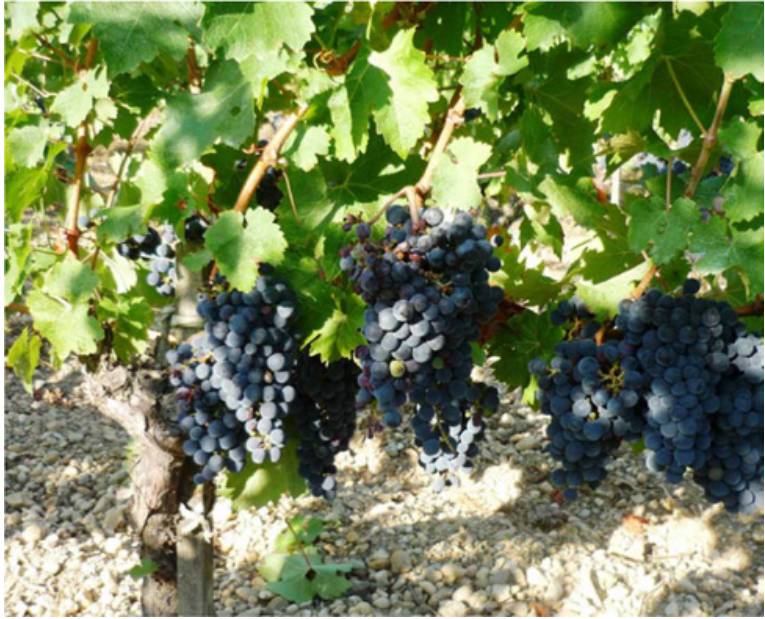

Photo 1. Cabernet-Sauvignon 1124, Chambre d'Agriculture Gironde.

D'ores et déjà des parcelles expérimentales ont été mises en place avec des présélections de clones de Grenache N et Merlot accumulant moins de sucres.

Récemment, des clones de Cabernet franc à fertilité modérée et grappes plus lâches ont été agréés. Ils traduisent ainsi la large diversité de ce vieux cépage.

Ces travaux s'accompagnent également de la sélection de clones pour des cépages orphelins. A titre d'exemple, le Castets N 1126 (cépage originaire du Bordelais), le Durif N ( « Petite Syrah » en Californie) 1130 et le Brun Argenté N 1164 (appelé également Vaccarèse dans les Côtes du Rhône) sont venus récemment abonder le Catalogue des clones.

Comment tout cela se traduit-il en termes de dynamique de plantations ? Il est encore trop tôt pour se prononcer car l'installation des vignes-mères prend quelques années avant que ce matériel soit disponible pour la filière. De plus, l'économie viticole étant encore fragilisée, les professionnels peuvent être réticents à prendre des risques avec des cépages peu connus du public et dont les performances technologiques demandent tout de même à être validée à plus grande échelle.

\section{Les cépages du Patrimoine Français, ou comment développer l'œnotourisme}

Le nombre de cépages autochtones du vignoble français présents dans la collection nationale est de 556 (source : T. Lacombe, INRA de Vassal). Chaque année les prospections menées dans les vieilles vignes conduisent à la découverte de cépages originaux qu'aucun grimoire n'avait décrits jusqu'alors.

Ces découvertes enrichissent la collection de l'INRA de Vassal et peuvent, parfois, apporter des éléments scientifiques sur les liens génétiques entre les cépages.

Une tendance se développe depuis quelques années à l'initiative des vignerons qui souhaitent revaloriser les anciens cépages de leur région.

Ainsi, des démarches visant à exploiter la diversité variétale pour lutter contre une forme de standardisation et proposer des vins différents aux consommateurs, 


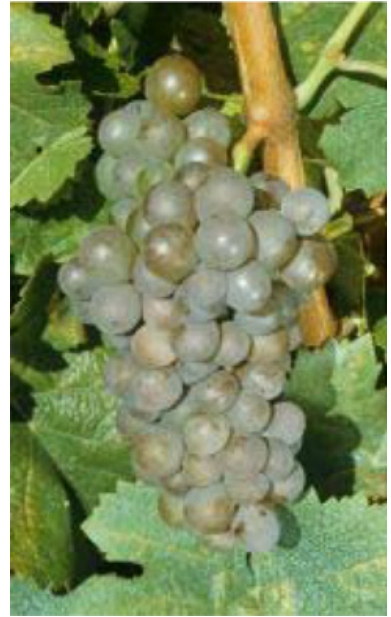

Photo 2. Grappe de Trousseau gris G (Chauché), Plantgrape.

permettent localement de développer l'œnotourisme tout en favorisant des circuits commerciaux courts.

Parmi ces initiatives « de niche », on peut citer :

- les Producteurs de Plaimont et l'emblématique classement en Monument Historique de la Parcelle de M. Pédebernade à Sarragachies (Gers) mais également les efforts pour redévelopper le Manseng noir $\mathrm{N}$ (lié génétiquement au Tannat selon les travaux de Boursiquot, Lacombe et al.),

- le Centre d'Ampélographie Alpine Pierre Galet (CAAPG).

En Savoie et au-delà dans tout le piémont alpin, le vignoble recèle de nombreux et anciens cépages autochtones. La valorisation de cette richesse est le fruit d'un travail besogneux de quelques vignerons.

Ainsi, le Persan $\mathrm{N}$ a retrouvé une notoriété qui dépasse la région. Mais, tout récemment, les Bia $\mathrm{B}$, Mècle $\mathrm{N}$, Sérènèze $\mathrm{N}$ ont été inscrits et classés au Catalogue. Le Robin N va suivre et le Chouchillon B, l'Onchette $\mathrm{N}$ ainsi que la Mondeuse grise $\mathrm{G}$ vont être bientôt présentés pour une inscription.

- Le Conservatoire du Vignoble Charentais.

Depuis une quinzaine d'années, le Conservatoire du Vignoble Charentais (CVC) s'est attaché à mener des prospections dans le vignoble traditionnel et sa périphérie sans oublier les îles d'Oléron, d'Aix ou de Ré.

La valorisation la plus emblématique est la réhabilitation du Trousseau gris G (appelé localement le Chauché gris).

Ce cépage est expérimenté actuellement pour la production de mistelles (pineau) ou pour la production d'eaux-de-vie. Il est trop tôt pour présager du succès que connaîtra ce cépage, mais son classement récent traduit la volonté professionnelle de revaloriser le patrimoine local.

\section{L'innovation avec les variétés étrangères, une variable d'ajustement vis-à-vis du changement climatique ?}

Depuis une bonne dizaine d'années, l'IFV a accéléré le processus dans ce sens en orientant ses recherches

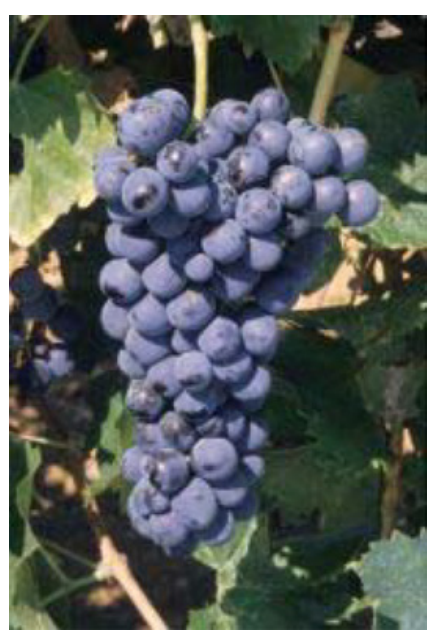

Photo 3. Grappe de Nero d'Avola 5 Calabrèse), Plantgrape.

sur les cépages du pourtour méditerranéen, c'est-à-dire grecs, italiens ou de la péninsule ibérique. Certains sont manifestement bien adaptés à la contrainte hydrique et aux températures estivales élevées et pourraient avoir un avenir en France : l'Agiorgitiko, le Xyno Mavro pour les rouges, l'Assyrtico, le Moscho filero, le Rhoditis Rs ou le Fiano pour les blancs.

D'ores et déjà, sont notamment inscrits au Catalogue, les cépages Touriga nacional, Nero d'Avola (Calabrese), Nebbiolo, Primitivo et Pinotage.

\section{L'innovation par la création variétale, la réponse durable à la réduction des intrants phytosanitaires ?}

Certes, il existe bien encore 20 hybrides (HPD) à usage de cuve dans le Catalogue National (ainsi que des variétés d'agrément et quelques variétés de table), mais la qualité des vins produits n'est pas suffisante pour les productions en AOP. Leur utilisation dans ce contexte est, de toute façon, actuellement proscrite par la réglementation européenne (bien que certaines appellations en France et en Allemagne aient inclus des variétés hybrides dans leur cahier des charges).

Il existe également des variétés déjà disponibles (et inscrites) en Allemagne ou en Suisse par exemple. Mais avant leur développement, il est cependant nécessaire de les expérimenter dans notre vignoble afin de vérifier leur comportement vis-à-vis des maladies cryptogamiques dans des conditions de pression différentes, et surtout d'évaluer la qualité du produit final.

Depuis une cinquantaine d'années, l'INRA effectue des travaux d'hybridations, principalement intraspécifiques. Ont été ainsi créés des variétés qui connaissent un réel succès, le Marselan N (Grenache N x Cabernet-Sauvignon N), le Caladoc (Grenache N x Cot $\mathrm{N}$ ), ou récemment le Cabestrel N (Cabernet-Sauvignon N x Mourvèdre N), inscrit au Catalogue en 2014.

La création de variétés issues de croisements inter spécifiques a été initiée dans les années 1980 par un chercheur de Montpellier, Alain Bouquet, et reprise et intensifiée par l'équipe INRA de Colmar au début des 


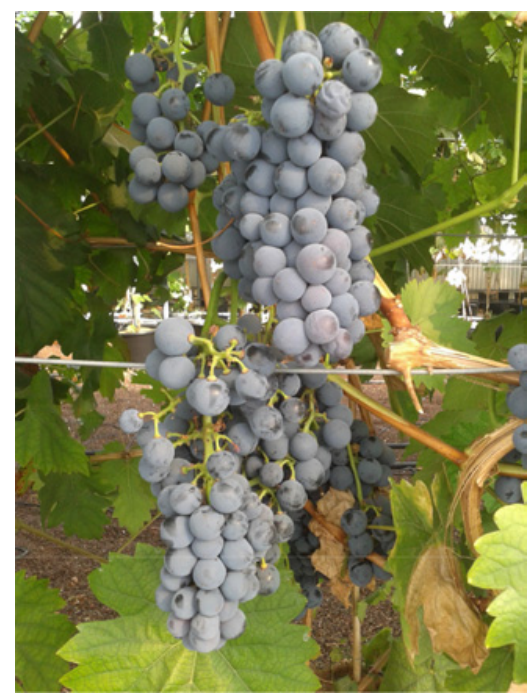

Photo 4. Génotype résistant, IFV.

années 2000. Il s'agit d'un programme appelé INRA Resdur (6).

La stratégie retenue par l'INRA est de ne proposer à la diffusion que des variétés à résistances polygéniques (au moins 2 QTL de résistance au mildiou et à l'oïdium) et dont les caractéristiques agronomiques, technologiques et organoleptiques ont été validées aux différents stades de la sélection.

Les premières inscriptions de ces variétés nouvelles sont attendues dans moins de 2 ans.

Désormais, l'IFV s'est également engagé dans la voie de la création variétale en suivant la stratégie de pyramidage de l'INRA. Après 50 ans de sélection clonale, cela traduit sinon un changement de paradigme, du moins une stratégie nouvelle rendue nécessaire par les limites de la sélection classique vis-à-vis de la lutte contre les maladies cryptogamiques.

L'objectif de ces deux instituts est, à moyen terme, d'associer durabilité des résistances avec adaptation pédoclimatique. Dans un deuxième temps, des « typicités particulières » seront recherchées, dans le but de mettre à la disposition de la viticulture des variétés durablement résistantes permettant de se rapprocher des productions représentatives d'une région viticole. Cela passe par une feuille de route donnée par les professionnels, « l'idéotype ». Il y a d'ores et déjà des demandes de la part de certains vignobles, pour les eaux-de-vie, pour les vins rosés, pour les vins effervescents et désormais pour le vignoble bordelais.

\section{Conclusions et perspectives}

Accompagner la viticulture française dans les différents challenges qu'elle doit relever, soutenir la compétitivité de la filière française, c'est donc mettre « tous les fers au feu » pour s'adapter aux enjeux de demain. Le paysage variétal est appelé à évoluer, mais cette évolution pressentie et inéluctable semble difficile à cerner au cours de ces prochaines années. En tout état de cause, l'innovation variétale constitue un levier majeur pour l'évolution nécessaire de ce secteur. Mais l'innovation ne réside pas seulement dans la création variétale, elle se matérialise aussi par l'intérêt potentiel de variétés de l'arc méditerranéen et, plus modestement par le regain d'intérêt accordé à nos variétés dites patrimoniales.

Cependant, l'appropriation par la profession et l'accueil que réserveront les consommateurs à ces différentes variétés est aujourd'hui difficile à prédire !

Vivement demain! Roll on Tomorrow!

\section{Références}

[1] J. Branas, 1974. Viticulture. Imp. Dehan, Montpellier, France, p.122-139, 659-689

[2] Site internet du catalogue des vignes cultivées en France : http://plantgrape.plantnet-pro ject.org/

[3] CTPS - GEVES, 2008. Règlement technique d'examen des variétés de vigne en vue de leur inscription au Catalogue Officiel des espèces et variétés de plantes cultivées en France

[4] CTPS, 2013. Règlement technique d'agrément des clones de vigne

[5] http://plantgrape.plantnet-project.org/ partenaires

[6] C. Schneider, 2014. Caractérisation et exploitation de résistances génétiques pour une viticulture à faibles intrants phytosanitaires. Présentation, Les rencontres de l'INRA, Salon de l'Agriculture 